\title{
THREE DISTINCT NON-HEREDITARY RADICALS WHICH COINCIDE WITH THE CLASSICAL RADICAL FOR RINGS WITH D.C.C.
}

\author{
Subrata Majumdar and Kalyan Kumar Dey ${ }^{1}$ \\ Department of Mathematics, Rajshahi University, Rajshahi-6205 \\ ${ }^{1}$ Corresponding author:kkdmath@yahoo.com
}

Received 05.01.2006 Accepted 06.09.2006

\begin{abstract}
Majumdar and Paul [3] introduced and studied a new radical $E$ defined as the upper radical determined by the class of all rings each of whose ideals is idempotent. In this paper the authors continue the study further and also study the join radical and the intersection radical (due to Leavitt) obtained from $E$ and the Jacobson radical $J$. These have been denoted by $E+J$ and $E J$ respectively. The radical and the semisimple rings corresponding to $E+J$ and $E J$ have been obtained. Both of these radicals coincide with the classical nil radical for Artinian rings. Important properties of these radicals and their position among the well-known special radicals have been investigated. It has been proved that $E, E J$ and $E+J$ are non-hereditary. It has also been proved as an independent result that the nil radical $N$ is not dual, i.e., $N \neq N_{\varphi}$.
\end{abstract}

\section{Introduction}

In [3] a radical $E$ was introduced and studied. In this paper a further study of $E$ has been made and certain unsolved problems regarding this radical have been settled. Moreover, two new radicals $R_{l}$ and $R_{2}$ obtained with the help of $E$ and $J$ using Leavitt's construction [4], have been studied. In particular it has been shown that $E, R_{1}$, and $R_{2}$ are three distinct non hereditary radicals each of which coincides with the classical radicals for rings with d.c.c. on left on right ideals.

Also it has been shown that $N$ is not a dual radical. Here $J$ is the Jacobson radical, $N$ the nil radical of Koethe. We have followed the terminology and the notations of Divinsky [1] except that we write radical for radical property.

*There is an unfortunate mistake in the title of [3]. It has been corrected in the reference. The first author expresses regret for the mistake.

2. Let $C$ denote the class of rings A such that, for every ideal $I$ of $A . I^{2}=I$, and let, $P$ denote the class of all prime rings. Let $D=C \cap P$. It was shown in [3] that $C$ and $D$ determine the same upper radical which we denote by $E$.

It was shown in Proposition 3.1 ([3]) that $L \subseteq E \subseteq G$, where $L$ and $G$ are the Levitzki radical and the Brown-McCoy radical respectively. Hence $E$ coincides with the classical radical rings with d.c.c. (see Theorem 13, [1]). It was also shown that 

(i) $\quad L \neq E$,
(ii) $G \neq E$,
(iii) $B_{\varphi} \subseteq E,(B$ is the Baer lower radical)
(iv) $E \subseteq V,(V$ is the upper radical determined by the class of all simple non-nilpotent rings)
(v) $E \not \subset N$, (its proof was not, however, complete)
(vi) $E$ and $J$ are independent of each other, $J$ being the Jacobson radical.

There we could not decide whether
(a) $E$ is hereditary,
(b) $B_{\varphi}<E$,
(c) $E<V$,
(d) $E<N$,

In this paper, we shall settle the first two of these questions. Also we shall give a complete proof of (v) (mentioned above) in [3].

We consider the following example:

\section{Example 2.1}

Let $F$ be a field and Let $A$ be a vector space on $F$ with a basis $\{\alpha, \beta\}$. Define multiplication in $A$ by the table:

\begin{tabular}{|c|c|c|}
\hline$\cdot$ & $\alpha$ & $\beta$ \\
\hline$\alpha$ & $\alpha$ & $\alpha$ \\
\hline$\beta$ & $\alpha$ & $\alpha$ \\
\hline
\end{tabular}

Then $A$ is a ring whose ideals are $A, F_{\alpha}$ and 0 . Since $A^{2}=F_{\alpha} \neq A$, and $\left(A / F_{\alpha}\right)^{2}=0 \neq A / F_{\alpha}, A$ is $E$ radical. But $F_{\alpha}$ is a simple ring with $\left(F_{\alpha}\right)^{2}=F_{\alpha}$. So $F_{\alpha}$ is $E$-semisimple. Hence $E$ is nonhereditary. Since $B_{\varphi}$ and $N$ are special radicals both of these are hereditary, and so, $E \neq B_{\varphi}$ and $E \neq N$. Since $A$ is $N$-semisimple, $E \not \subset N$.

Thus we have thus proved:

\section{Theorem 2.1}
(i) $E$ is non-hereditary,
(ii) $B_{\varphi}<E$,
(iii) $E \not \subset N$.

This theorem answers the questions (a) and (b) of section 2 in the affirmative and gives a complete proof of (v).

3. In this section we digress and prove that the nil radical is not dual.

\section{Theorem 3.1}

$N<N_{\varphi}$, i.e. $N$ is not a dual radical. 
Proof: Let $A$ be the ring of all rational numbers of the form $2 x /(2 y+1)$ where $x$ and $y$ are integers with $(x, 2 y+1)=1$ (see [1], Example 10). $A$ is obviously $N$-semisimple.

Let $I$ be any non-zero ideal of $A$ and Let $n^{\prime}$ be the smallest positive integer such that $2^{\mathrm{n}^{\prime}} x /(2 y+$ $1) \in I$, for some odd integer $x$. Let $i=2^{n^{\prime}}(2 z+1) /(2 y+1) \in I$, for some integers $y$ and $z$ such that $(2 z+$ $1,2 y+1)=1$.

We note that $(2 z+1) 2^{\mathrm{n}^{\prime}}=(2 \mathrm{y}+1) i \in I$ and so, $2 \cdot 2^{\mathrm{n}^{\prime}}=[2 /(2 z+1)](2 z+1) \cdot 2^{\mathrm{n}^{\prime}} \in I$. Since $(2,2 z+1)=1$ there exist integers $\lambda$ and $\mu$ such that $2 \lambda+(2 z+1) \mu=1$ and so $2^{n^{\prime}}=2 \lambda \cdot 2^{n^{\prime}}+(2 z+1) \mu \cdot 2^{n^{\prime}} \in I$. Therefore, $2^{\mathrm{n}^{\prime}} A \subseteq I$.

Also, for each $i \in I$

$$
\begin{aligned}
i & =2^{\mathrm{n}^{\prime}} /(2 u+1), \text { for some integers } u \text { and } v \text { with }(u, 2 v+1)=1 . \\
& =2^{\mathrm{n}^{\prime}-1} \cdot 2 u /(2 v+1) \in 2^{\mathrm{n}^{\prime}-1} \cdot A .
\end{aligned}
$$

Thus, $2^{n^{\prime}} \cdot A \subseteq I \subseteq 2^{n^{\prime}-1} \cdot A$.

Also, for each non-negative integer $n, 2^{n} A$ is an ideal of $A$. Therefore, it follows that $A$ is subdirectly reducible and that every non-trivial homomorphic image of $A$ is nil. Thus, $A$ cannot be mapped homomorphically onto a subdirectly irreducible ring with a nil semisimple heart. So $A$ is $N_{\varphi}$ radical.

Hence $N \neq N_{\varphi}$ and the proof is complete.

\section{Radicals $E J$ and $E+J$}

It has been shown in [3] the radical $E$ and $J$ are independent of each other, i.e., neither of $E$ and $J$ contains the other. Hence both $E \cup J$ and $E \cap J$ are distinct and are different from both $E$ and $J$. Thus the intersection radical $E J$ and the join radical, $L_{E \cup J}$, the lower radical determined by $E \cup J$, are two distinct radicals different from both $E$ and $J$. We denote $E J$ by $R_{1}$ or $E J$ and $L_{E \cup J}$ by $E+J$ or $R_{2}$. We first note the following:

(i) $E J$ and $E+J$ coincide with the classical nil radical for rings with d.c.c. on left or right ideals.

Since both $E$ and $J$ lie between $D$ and $T$, where $D$ is the lower radical determined by all nilpotent rings which are nil radicals of rings with d.c.c. on left ideals, and $T$ is the upper radical determined by all matrix rings over division rings, it follows that both $E J$ and $E+J$ too lie between $D$ and $T$. Hence by Theorem 13 of [1] both $E J$ and $E+J$ coincide with the classical nil radical.

\section{2. $E J$ and $E+J$ are non-hereditary}

The ring $A$ in example 2.1 has d.c.c on left ideals. Hence the radicals $E J$ and the $E+J$ radicals of $A$ and $F_{\alpha}$ are the same as the $E=$ radicals of $A$ and $F_{\alpha}$ respectively. It thus follows that just like $E$, both $E J$ and $E+J$ are non-hereditary.

The radical rings and the semisimple rings with respect to the radicals $E J$ and $E+J$ are described below: 


\section{1) EJ-radical rings}

It is clear from the definition of $E J$ the radical rings are precisely the rings which cannot be mapped homomorphically onto a non-zero ring in $C_{P}$ where $P$ is the class of all primitive rings. Thus, the $E J$-radical rings are precisely those whose non-zero homomorphic images are neither primitive nor have each ideal idempotent.

\section{2) $E J$-semisimple rings}

It follows from the construction of the intersection radical $(E J=E \cap J)$ of a ring $A$ (see Leavitt [2]) that $A$ is $E J$-semisimple if and only if $E(A) \cap J(A)=0$.

To have an idea about the $(E+J)$-radical of $A$, we need a few results due to Majumdar ([3]).

It has been shown by Majumdar and Saha ([4]) that, if a class $C$ of rings satisfies certain properties called $P_{1}$ and $P_{2}$ which are described below, then the lower radical $L_{c}$ and the corresponding radical $L_{c}(A)$ for a ring $A$ can be expressed in a way similar to that for the radical $B(A)$ for $A$, where $B$ is the Baer lower radical. Because we shall use this fact, we state here the relevant results without proof. We start with two definitions and a notation:

Definition: A non-empty class $C$ of rings is said to have property $P$ if $C$ is homomorphically closed, and is said to have property $P_{2}$ if for every ring $A$ and every nonzero ideal $I$ of $A, A$ has no non-zero $C$-ideal implies $I$ has no non-zero $C$-ideals.

Notation: Let $C$ be a non empty homomorphically closed class of ring, and let $A$ be a ring. Let $A_{\tau}$ be the sum of all $C$-ideals of $A$. For an ordinal $\beta>2$ and not a limit ordinal, define $A_{\beta}$ to be the ideals of $A_{\beta} / A_{\beta-1}$ such that $A_{\beta} / A_{\beta-1}$ is the sum of all $C$-ideals of all $C$-ideals. of $A / A_{\tau}$ and if $\beta<2$ is a limit ordinal, define $A_{\beta}=\cup A_{\alpha}(\alpha<\beta)$ Thus $A_{\alpha}$ exists for each ordinal $\alpha$. Then there is a smallest ordinal $\tau$ such that $A_{\tau+1}=A_{\tau}$. We denote $A_{\tau}$ by $A_{C}$.

We next state the useful results:

\section{(a) Theorem 4.1}

Let $C$ be a non-empty class of rings satisfying conditions $P_{1}$ and let $A$ be a ring. Then $A$ is the intersection of all ideals $Q_{\mathrm{i}}$ of $A$ such that $A / Q_{i}$ has no non-zero $C$-ideals.

\section{(b) Theorem 4.2 (see [4])}

Let $C$ be a non-empty class of rings satisfying conditions $P_{1}$ and $P_{2}$. Then the class $R$ of all rings such that $A=A_{c}$ for some rings $A$, is a radical.

Also $R_{\mathrm{c}}(B)=B_{\mathrm{c}}$ for every ring $B$ with properties $P_{1}$ and $P_{2}$.

\section{(c) Theorem 4.3 (see [4])}

Let $C$ a non-empty class of rings satisfying conditions $P_{1}$ and $P_{2}$, Then $R_{c}=L_{c}$ is the lower radical determined by $C$. 


\section{(d) Theorem 4.4}

If $C$ is a non-empty class of rings with conditions $P_{1}$ and $P_{2}$, then a ring $A$ is $L_{\mathrm{c}}$-semisimple if and only if $A$ has no zero $C$-ideals.

The last theorem is a direct consequence of Theorem 4.2, Theorem 4.3 and the definition of $A_{c}$.

We are now ready to discuss the $(E+J)$-radical rings and the $(E+J)$-semisimple rings.

\section{(3) $(E+J)$-radical rings}

We readily see that if $R_{1}$ and $R_{2}$ are two radicals, then $R_{1} \cup R_{2}=R_{R 1 \cup R 2}$ and so, the $\left(L_{R 1 \cup R 2}\right)$-radical of a ring $A$ is $A_{R l \cup R 2}$. Thus the $(E+J)$-radical of a $\operatorname{ring} A$ is $A_{E \cup J}$ and so, the $(E+J)$-radical rings are precisely the rings $A$ such that $A=\mathrm{A}_{E \cup J}$.

\section{(4) $(E+J)$-semisimple rings}

By Corollary 4.4, a ring $A$ is $L_{R 1 \cup R 2}$ semisimple if and only if $A$ has no non-zero $R_{1} \cup R_{2}$ ideals i.e., if and only if $A$ is both $R_{1}$-semisimple and $R_{2}$-semisimple. Hence we have.

The $(E+J)$-semisimple ring are precisely the rings $A$ which are both $E$-semisimple and $J$ semisimple i.e., $S(E+J)=S(E) \cap S(J)=$ The class of all those subdirect sums of primitive rings each non-zero ideal of which can be mapped onto a nonzero ring in $D$. (or equivalently in $C$ ). Here $S(R)$ denotes the semisimple class of a radical $R$.

\section{REFERENCES}

[1] Divinsky N. J., Rings and Radicals, University of Toronto Press, (1965).

[2] Leavitt W.G., Sets of radical classes, Publications Mathematicae, 14 (1967), 321-325

[3] Majumdar S. and Paul A. C., A non-hereditary (?) radical which coincides with the classical radical for rings with d.c.c., Ganit (J. Bangladesh Math Soc), 1(2) (1981), 85 - 89.

[4] Majumdar S. and Shaha S. K., On the lower radical determined by a class of rings, Ganit (J. Bangladesh Math Soc), 9(1) (1989), 39 - 44. 